Maltese population and that of other studies. Clinically, one of the most consistent findings among immigrant ethnic groups in Western Europe is an increase in the incidence rate of psychosis. A recent meta-analysis has confirmed that migration is a risk factor for schizophrenia that cannot be solely explained by selection (Cantro-Grae \& Shelton, 2005; see also Veiling et al, 2008).

\section{Limitations}

Our study excluded those suffering from a form of psychotic illness who were treated in the community. Nonetheless, since the Maltese National Health Service does not have an urgent care and home management team, we can expect that most patients suffering from a first episode of psychosis would be admitted to hospital.

The authors acknowledge that this study may suffer from the ecological fallacy: the sample population may not be truly representative of the target population. So the results should be interpreted with care.

Another limitation was that we did not include patients admitted to a private hospital, but, when the study was carried out, the private sector had no admitting units specifically for psychotic patients.

Data were solely collected from medical files; that is, with no interviews of the admitting doctors or patients themselves.

\section{Clinical implications}

The results of this study highlight that the incidence of psychosis is variable. In Malta, as in other countries, urbanisation, low socio-economic status and immigration are potential risk factors in the aetiology of psychosis. Higher educational attainment may be a protective factor. Awareness of this by clinicians and service providers can lead to better provision and planning of services.

\section{Acknowledgements}

We would like to thank Dr Neville Calleja MD MSc for his input of the statistical data.

\section{References}

Camilleri, F. (2001) A Day in Her Life: Insights into the Social and Economic Contribution of Maltese Women. Commission for the Advancement of Women, Ministry of Social Policy, Malta.

Cantro-Grae, E. \& Shelton, J. P. (2005) Schizophrenia and migration: a meta-analysis review. American Journal of Psychiatry, 162, 12-24.

Haukka, J., Suvisaari, J., Varilo, T., et al (2001) Regional variation in the incidence of schizophrenia in Finland: a study of birth cohorts born from 1950-1969. Psychological Medicine, 31, 1045-1053.

Kendler, K. S., Gallagher, T. J., Abelson, J. M., et al (1996) Lifetime prevalence, demographic risk factors, and diagnostic validity of nonaffective psychosis as assessed in a US community sample: the National Comorbidity Survey. Archives of General Psychiatry, 53, 1022-1031.

Kirkbride, J. B., Fearon, P., Morgan, C., et al (2006) Heterogeneity in incidence rates of schizophrenia and other psychotic syndromes. Archives of General Psychiatry, 63, 250-258.

Marcelis, M., Navarro-Mateu, F., Murray, R., et al (1998) Urbanization and psychosis: a study of 1942-1978 birth cohorts in the Netherlands. Psychological Medicine, 28, 871-879.

Marcelis, M., Takei, N. \& Van Os, J. (1999) Urbanisation and risk for schizophrenia: does the effect operate before or around the time of illness onset? Psychological Medicine, 29, 1197-1203.

Menezes, P. R., Scazufca, M., Busatto, G. F., et al (2007) Incidence of first-contact psychosis in Sao Paulo, Brazil. British Journal of Psychiatry, 191, 102-106.

Sundquist, K., Frank, G. \& Sundquist, J. (2004) Urbanisation and incidence of psychosis and depression: follow-up study of 4.4 million women and men in Sweden. British Journal of Psychiatry, 184, 293-298.

Van Os, J. (2004) Does the urban environment cause psychosis? British Journal of Psychiatry, 184, 287-288.

Van Os, J., Driessen, G., Gunther, N., et al (2000) Neighbourhood variation in incidence of schizophrenia. Evidence for person-environment interaction. British Journal of Psychiatry, 176, 243-248.

Veiling, W., Susser, E., Van Os, J., et al (2008) Ethnic density of neighbourhoods and incidence of psychotic disorders among immigrants. American Journal of Psychiatry, 165, 66-73.

\title{
Collaboration between traditional healers and psychiatrists in Sudan
}

\author{
E. A. Sorketti, ${ }^{1}$ N. Z. Zuraida ${ }^{2}$ and M. H. Habil ${ }^{3}$
}

${ }^{1}$ Psychiatrist, Department of Psychological Medicine, Faculty of Medicine, University of Malaya, Kuala Lumpur, Malaysia, email ehabsorketti@yahoo.com; ${ }^{2}$ Consultant Psychiatrist, Department of Psychological Medicine, Faculty of Medicine, University of Malaya; ${ }^{3}$ Head, Department of Psychological Medicine, Faculty of Medicine, University of Malaya$$
\text { he importance of traditional healing in low- and }
$$
middle-income countries cannot be underestimated. It is generally perceived as part of the prevailing belief system and traditional healers are often seen as the primary agents for psychosocial problems in these countries; estimates of their service share range from $45 \%$ to $60 \%$ (World Health Organization, 1992). The World Health Organization (2000) estimated that $80 \%$ of people living in rural areas in low- and middleincome countries depend on traditional medicine for their health needs.
In Sudan, a country with a mixed Arab/African culture, traditional healing is the most common method of treating people with mental illness, mostly because it is usually far cheaper than medical treatment (Elsafi \& Baasher, 1981-94) but also because of the inaccessibility of medical services and lack of awareness among the population. Baasher (1994) suggested that the holistic approach of traditional healing may lead to long-term stability of health. There is, though, no regulation of traditional healers and consequently many cases of abuse have been reported. Sorketti (2009) mentioned that the treatment of severe mental disorders is not available at 
Box 1 Glossary

Bakhara. Special verses written on a sheet of paper that is burnt and the smoke used

Khalwa. Preschool education in traditional healer centres Kogour. Special type of healing in African culture

Mehaya. Verses written on a sheet of paper that is dissolved in water and drunk

Rogya. Reading the words of God on a person with mental illness

Sheikh. Holy man

Talasim. Figures and letters and special drawings that have certain meanings

Zar. Ceremony for a person possessed by spirits

The term sheikh is equivalent to traditional healer in this text

primary care level in Sudan, which is why traditional healers are often used for the provision of mental health services.

Generally, traditional healers in Sudan can be divided into two distinct groups: religious healers, influenced by Islamic and Arab culture, such as traditional Koranic healers and Sufi healers; and non-religious healers, influenced by African culture, such as practitioners of zar, talasim and kogour (see Box 1 for glossary). The religious healers in turn may be subdivided into two groups. The first group uses only Koranic treatment, derived from certain verses. This involves reading and listening to the Koran with the active participation of the patient (Bali, 1992). The success of treatment depends on the reliability of the healer and the degree of his or her belief, in addition to the conviction of the patient and his or her belief in the Koran as a source of treatment. The second group uses a combination of both Koran and talasim. The types of talasim used are mainly squares filled with symbolic letters which have a hidden spiritual dimension conceived only by the sheikhs (holy men). They contain the 99 attributes (names) of God and some other words from ancient divine books. Healers in this subgroup are influential decision makers at the individual, family and community level. They are respected not only by their followers but also by government officials and politicians.

Elsorayi (1985) stated that kogour is a typical African practice found in the south of Sudan, where African culture dominates. It is used by healers who claim to have supernatural powers; it deals with 'souls' that affect the body. Such healers use their power to cure disease and to solve other problems, such as the control of rain.

Mohammed (1989) suggested that zar came to Sudan from Ethiopia. It is based on the assumption that supernatural agents or spirits possess a person and may generate physical and psychological disorders. The zar concept of possession is based on the idea that the spirit makes certain demands that should be fulfilled by the patient or relatives; otherwise this spirit may cause trouble for them all. Zar is the dominance of the evil soul over the human being, with the intention of hurting the person. Zar is common among Muslims as well as Christians.

\section{Study objectives}

Our general objectives were to study and understand the traditional healers' beliefs and practices in relation to people with mental illness in Sudan. We also sought to assess the possibilities for collaboration between traditional healers and psychiatrists in Sudan.

\section{Method}

We conducted a descriptive cross-sectional study of traditional healers' attitudes, beliefs and practices in relation to people with mental illness. The study drew from randomly selected famous traditional healers' centres in Sudan.

Over 3 months (June-August 2009), 30 traditional healers from ten traditional healers' centres were randomly selected. They were approached individually and their consent was obtained before the principal investigator interviewed them with a 15-item structured questionnaire that covered:

O age

O education level

O occupation

O place of work

previous Job

O length of practice treating people with a mental illness

O how the treatment of people with a mental illness had been learnt

method of diagnosis

o methods of treatment

O length of time it typically took patients to respond to treatment

O length of time for which patients with mental illness were generally kept in the centre

o how many patients with mental illness were seen every day

what the healer thought about medical treatment for mental illness

o what the healer thought about patients who took traditional treatment and medical treatment at the same time

O whether it was possible to collaborate over medical treatment and traditional treatment, and if so, how.

Ethical approval was obtained from the Research Ethical Committee of the Sudanese Ministry of Health before the start of the study. Data were analysed using SPSS version 16.

\section{Results}

Twenty-eight traditional healers agreed to be interviewed (a 93\% response rate). They were aged 38-75 years. Ten of them (36\%) had received no formal training in their practice but had learnt it only in their traditional healer centres. Six $(21 \%)$ of them had been to formal primary school, seven (25\%) to secondary school and five (18\%) to university. Eleven (39\%) were farmers, nine (32\%) were teachers in the traditional centres, four (14\%) were traders and another four (14\%) were previously employed in the government. The number of years of practice of the healers (specifically in relation to treating mental illness) ranged from 10 to 50 years. They had learnt the methods of treatment from their parents and other healers.

Half of them followed certain criteria to diagnose mental illness. They divided mental illness into that which needs the intervention of a traditional healer, such as possession by evil spirits, jinn or shaitan, and that which needs a doctor's 
Table 1 Effect of the traditional healers' education level on their opinion regarding medical treatment for mental illness

\begin{tabular}{lccr}
$\begin{array}{l}\text { Education level of the } \\
\text { traditional healer }\end{array}$ & \multicolumn{3}{l}{$\begin{array}{l}\text { Traditional healer's opinion on } \\
\text { medical treatment }\end{array}$} \\
\cline { 2 - 4 } & Useful & Not useful & Total \\
Khalwa (see glossary, Box 1) & 2 & 8 & 10 \\
Primary school & 4 & 2 & 6 \\
Secondary school & 4 & 3 & 7 \\
University and above & 5 & 0 & 5 \\
Total & 15 & 13 & 28
\end{tabular}

Pearson $\chi^{2}=9.314$, d.f. $=3(P=0.025)$; likelihood ratio $=11.466$, d.f. $=3$ $(P=0.009)$.

intervention, such as some cases of acute fever or epilepsy. The other half of the healers instead looked at the overall symptoms of the patients. Those who had features of anxiety, mild depression, somatoform disorders or adjustment disorders were considered mild cases of mental illness, while those who had lost their sense of reality and who were severely agitated or aggressive or socially withdrawn and neglecting their personal hygiene and were unable to function were considered psychotic and to have severe mental illness.

According to the traditional healers, patients could take a few weeks, months or even years to get well.

Thirteen (46\%) of the healers reviewed on average three to five patients per day, while seven (25\%) saw five to ten patients a day and the remaining eight (29\%) saw fewer than three patients a day.

Fifteen (54\%) of the healers believed that psychiatric medication was useful for treating mental illness and they believed that combining traditional treatment and psychiatric medication could be useful. The other 13 (46\%) did not believe in medical treatment and thought that psychiatric medication was not useful; neither did they see any value in combining medical and traditional treatment. Belief in the value of psychiatric medication and modern psychiatric management was related to the educational level of the traditional healer: the more years of formal education the healer had received, the stronger was the belief in modern methods of management and the use of psychiatric medication for treating people with mental illness (Table 1) $(P=0.025)$.

A large majority $(25$, or $89 \%$ ) of the traditional healers were ready to collaborate with psychiatrists and mental health services (this was not associated with educational level). Only three $(11 \%)$ would refuse to collaborate. The traditional healers suggested three possible methods of collaboration: 56\% (14 out of the 25 healers who agreed with the idea of collaboration) suggested that they could refer some patients to a psychiatrist (while continuing with their traditional treatment) or for medical investigations; $32 \%$ (eight healers) suggested that psychiatrists or doctors trained in the management of people with mental illness could visit the traditional healer centres regularly to manage patients and give them medication; and the remaining $12 \%$ (three healers) would prefer joint clinics with a psychiatrist to manage people with mental illness.

The healers used similar methods of management to treat people with mental illness, such as mehaya, bakhara and rogya (see Box 1), controlling food intake and putting the patient in chains in the initial phase of management.

\section{Discussion}

Traditional healers in Sudan perform many valuable services. Nevertheless, traditional healing is not formally institutionalised, as there is no responsible government body to supervise delivery of these services. Ahmed et al (1999) stated that traditional healers act as family counsellors in critical life events such as building a house, marriage and naming a newborn child, and may have both judicial and religious functions. They often act as an agent between the physical and spiritual worlds. Thus traditional healers, in the people's eyes, are true representatives of spiritual power (Wad Daifalla, 1975).

The present study suggests that collaboration between traditional healers and medical services in the treatment of people with mental illness is of great importance, because most people who have a mental illness go to traditional healers first or alternate between healers and doctors, thereby wasting resources. We could at the least make use of traditional healer centres as Western-type community psychiatric centres. Peltzer \& Machleidt (1992) studied traditional healing methods in many African societies, as well as the bio-psychosocial therapeutic models in a traditional African setting (in Malawi). In particular, they looked at the therapeutic setting for schizophrenia in three traditional centres in terms of organisation, environment, culture, family and follow-up, and compared it with the Western model of psychiatric practice. They concluded that the traditional approach was in a number of ways superior to the Western model.

More research on the role of traditional healers in relation to people with mental illnesses is needed. Nonetheless, we should try to convince traditional healers of the benefits and the importance of giving modern psychiatric medications to their patients, under a psychiatrist's supervision. At the same time, they can continue the beneficial traditional methods of treatment that do not cause any harm to the patient. The late Professor E. L. Tigani el Mahi, the father of African psychiatry, stressed that our attitudes to religious healers should aim to encourage good-quality practice while trying to end harmful or faulty methods (Elsafi \& Baasher, 1981-94). In the present study, $89 \%$ of the traditional healers would accept collaboration with psychiatrists, and 54\% believed that modern psychiatric medications are useful for treating people with mental illness. In fact, in Sudan over more than 30 years, a symbiotic working relationship has been developed with faith healers working in the area, as part of community-based mental health programmes. There was initially a great deal of resistance by the faith healers, who looked on the mental health professionals as competitors, but a non-confrontational approach brought home the message that there are indeed areas, for example emotional disorders, where collaboration between the two is possible (World Health Organization, 2000).

\section{Limitations of the study}

In this study we included only traditional healers. We need to involve psychiatrists and interview them as well to get their opinions about traditional healing practices and collaboration.

\section{Recommendations and clinical implications}

$O$ It is vital to establish channels of collaboration and common understandings between traditional healers and mental health professionals in Sudan and other African 
countries where a majority of people with mental illness consult traditional healers first.

O If psychiatrists are able to collaborate with traditional healers, the latter could help in the early detection and early management of mental illness, with the prospect of better outcomes.

O Collaboration between psychiatrists and traditional healers could help to end harmful methods of practice by the traditional healers, such as isolating patients in an unhealthy, non-hygienic environment, depriving patients of nutritional food, beating patients, misdiagnosis and mismanagement.

O Collaboration could help to improve community awareness and decrease the stigma of mental illness.

O The traditional healer centres could be used as the basis for community rehabilitation facilities for people with mental illness.

O Improving the education level of traditional healers might enable them to have a better understanding of mental illness and of the benefits of modern medications (see also Table 1). Organising educational seminars and workshops for them might be helpful in this area.

\section{Acknowledgement}

The idea for conducting this research came to mind after many visits in 2007-09 to traditional healers' centres in Sudan and seeing the miserable conditions for patients. Also on these visits were: the WHO regional adviser for mental health in the Eastern Mediterranean Region, Dr Mohammed Tagy Yasamy; the director and the former director of the Institute of
Psychiatry at Oslo University, Professors Lars Lien and Edvard Hauff; the director of the SINTIF Research Institute in Norway, Professor Aren Aida; and, from the University of Malaya, Malaysia, Professor Hussian Habil, head of the Department of Psychiatry, and Professor Nor Zuriada.

\section{References}

Ahmed, I. M., Bremer, J. J., Magzoub, M. M. E., et al (1999) Characteristics of visitors to traditional healers in central Sudan. Eastern Mediterranean Health Journal, 5, 79-85.

Baasher T. (1994) Promotion and development of traditional medicine. WHO Magazine, 68

Bali W. (1992) Elsarim elbattar fi eltsadi llisahra walashrar. [The Final Word Against Magicians and Witches.] Elsahaba Library Press.

Elsafi, A. \& Baasher, T. A. (1981-94) Selected Essays of Tigani El Mahi. University of Khartoum Press.

Elsorayi, A. (1985) Ten Years in the South of Sudan. Kuwait University Press.

Mohammed, F. (1989) El-zar el-sudani. A Survey of Attitudes, Beliefs and Social Perspective Towards Zar in the Sudan. Khartoum University Press.

Peltzer, K. \& Machleidt, W. (1992) A traditional (African) approach towards the therapy of schizophrenia and its comparison with Western models. International Journal for Therapeutic and Supportive Organizations, 13, 203-221.

Sorketti, E. A. (2009) Sudan's national mental health programme and burden of mental illness. International Psychiatry, 6, 16-18.

Wad Daifalla, F. Y. T. (1975) Reviews by Wad Daifalla. Khartoum University Press (in Arabic).

World Health Organization (1992) The Promotion and Development of Traditional Medicine, Technical Report Series No. 622. WHO.

World Health Organization (2000) Promoting the Role of Traditional Medicine in Health Systems. Strategy for the African Region, Resolution AF\RC50\R3. WHO.

\section{SPECIAL PAPER}

\section{Reproductive risk: its role in maternal mental health}

\section{Carol Henshaw}

Consultant in Perinatal Mental Health, Liverpool Women's Hospital, Liverpool, UK, email chenshaw@doctors.org.uk

Eor or many women, pregnancy and childbirth are not without substantial risk in terms of new-onset, recurrent or existing mental disorder. This has consequences not only in terms of poor maternal mental health but also in terms of increased pregnancy- and delivery-related morbidity and can have a significant negative impact on the well-being of the fetus or neonate. New-onset disorders such as postnatal depression and puerperal psychosis have been recognised for some considerable time but it is also becoming apparent that, with the exception of anorexia nervosa, severe intellectual disability and possibly schizophrenia, conception rates among women with all types of mental disorder are the same as those in the general population. In high-income countries, the widespread use of atypical antipsychotics, most of which do not impair reproductive function, may lead to increased conception rates in women with schizophrenia. In addition, pregnancy and childbirth are multifactorial stressors which may render women with previous mental disorders vulnerable to a recurrence. Hence it is no surprise that studies in urban, low-income and ethnically diverse populations in the USA estimate that around a third of pregnant women are suffering from a mental disorder when substance misuse is included (e.g. Kim et al, 2006).

\section{Mood disorders}

Women with mood disorders are those at highest risk of a recurrence in relation to childbirth. Up to two-thirds of women with bipolar disorder experience an episode in the immediate postpartum period. Those with a first-degree relative with a history of puerperal relapse are particularly vulnerable. The risk remains high even if the woman has been well during pregnancy and for the 2 years before she became pregnant and despite her living in good social circumstances with 